\title{
A Multicast Extension to HMIPv6 with Efficient MAP Selection
}

\author{
Ming-Han Liu and Chun-Chuan Yang \\ Multimedia and Communications Laboratory \\ Department of Computer Science and Information Engineering \\ National Chi Nan University, Taiwan, R.O.C. \\ ccyang@csie.ncnu.edu.tw
}

\begin{abstract}
By considering the moving area of the mobile hosts, a more efficient MAP (Mobile Anchor Point) selection scheme is proposed in the paper to improve the default MAP selection algorithm in Hierarchical Mobile IPv6 (HMIPv6). Moreover, given that few research papers discussing the multicast extension of HMIPv6, an efficient multicast extension of HMIPv6 integrating the proposed MAP selection algorithm is also proposed. The proposed multicast scheme is basically an improvement of Remote Subscription (RS) applied in HMIPv6. Simulation study shows that the average number of control messages for multicast tree maintenance is significantly smaller than that of the original RS scheme, which means the multicast tree constructed by the proposed scheme is relatively stable. Moreover, the average delivery cost of proposed multicast scheme is close to that of $R S$.
\end{abstract}

Keywords: HMIPv6, Mobility Management, Multicast

\section{INTRODUCTION}

Mobile IPv6 (MIPv6) was officially standardized as RFC3775 [1] in 2004 by Internet Engineering Task Force (IETF) to realize seamless IP communications. However, communications quality in MIPv6 degrades because of the high signaling overhead and long signaling latency. To reduce the signaling cost and enhance seamless handoff, a hierarchical architecture for supporting local registrationHierarchical Mobile IPv6 (HMIPv6) [2] was proposed. In HMIPv6, a new entity that acts as a local Home Agent (HA) called Mobile Anchor Point (MAP) is introduced. A mobile host $(M H)$ sends Binding Update $(B U)$ to MAP rather than its HA that is typically far away, so the signaling overhead and handoff latency can be alleviated. To provide efficient local mobility management, a multilevel hierarchy of MAPs in HMIPv6 is recommended.

The default MAP selection algorithm specified in the draft of HMIPv6 is based on selecting the MAP that is most distant to avoid frequent re-registrations, which means the choice of a more distant MAP would reduce the probability of having to change a MAP and informing all correspondent nodes and the HA. However, the default MAP selection algorithm apparently results in heavy load in higher MAPs as well as a longer tunneling path between selected MAP and MH. By considering the moving area of a mobile host, a more efficient

This work was supported in part by the National Science Council, Taiwan, R.O.C., under grant NSC 93-2219-E-260-004
MAP selection scheme is proposed in the paper.

To support multicast service in MIP, two approaches were proposed in RFC3775, namely bi-directional tunneling (BT) and remote subscription $(R S)$. In BT, a mobile host must subscribe to the groups of interest through the HA. When the mobile host is away from home, the multicast datagrams are first routed to the HA, from where the datagrams are encapsulated in a unicast packet destined to the $\mathrm{MH}$ via tunneling. This approach handles source mobility as well as recipient mobility, and in fact hides host mobility from all other members of the group.

On the other hand, RS requires a mobile host to re-subscribe to the joined group by sending out a Multicast Listener Discovery $(M L D v 2)$ report message [3] on the newly visited foreign network while roaming. In this way, the multicast delivery path in RS is always the shortest path tree from the multicast source to all the receivers. While having the advantages of simple implementation and efficient delivery, RS suffers from serious packet losses as well as the higher signaling cost for multicast tree reconstruction due to using join-and-leave mechanism for mobile hosts to participate in groups of interest.

Quite a few techniques have been proposed on the basis of Mobile IP to provide multicasting with mobility functions, such as MoM (Mobile Multicast) [4], RBMoM (Range-Based Mobile Multicast Protocol) [5], MMA (Multicast by Multicast Agent) [6, 7], and MMROP (Mobile Multicast with Route Optimization) [8], etc. Multicast-related research for HMIPv6 or other micro-mobility protocols has received less attention in the literature. In addition to the proposed MAP selection scheme, a multicast extension to HMIPv6 integrating that MAP selection algorithm is also proposed in the paper. As will be shown in the section of simulation study, the performance of proposed MAP selection scheme as well as the multicast extension is better than their counterparts.

The remainder of the paper is organized as follows. First of all, we make a brief survey for HMIPv6 as well as some existing MAP selection schemes in section II. The proposed MAP selection scheme is presented in section III. The multicast extension integrating the MAP selection scheme is proposed in section IV. Simulation environment and results for performance evaluation are presented in section V. Finally, we conclude the paper in section VI. 


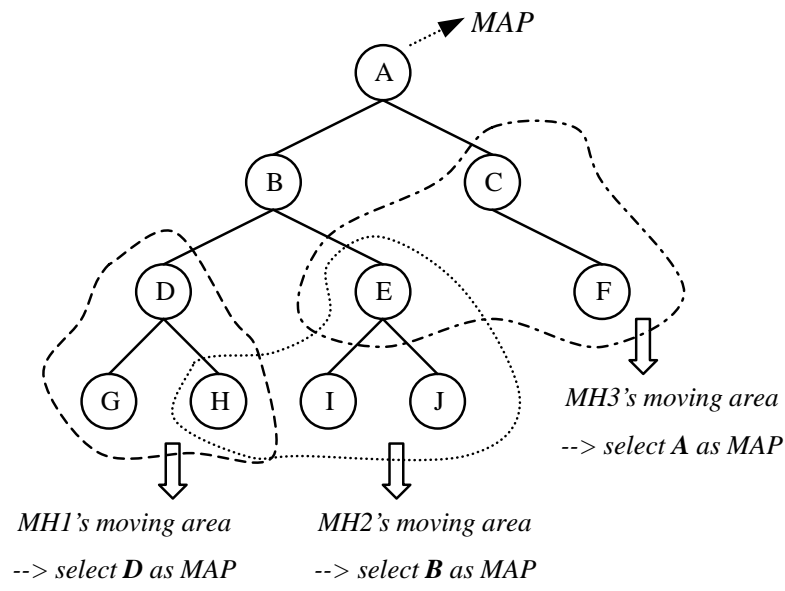

Figure 1. Moving Area-based MAP selection

\section{RELATED WORK}

As mentioned in section I, HMIPv6 was proposed to reduce signaling cost and latency in MIPv6 by localizing registration. Thus, the idea of Mobile Anchor Point (MAP), which acting as the local HA, is introduced in the foreign domain, and a multilevel hierarchy of MAPs in HMIPv6 is recommended. The Access Router (AR) that in charge of a subnet in the HMIPv6 network can be equipped with MAP function. Each MAP in the hierarchy sends out MAP options periodically to its downstream MAPs. The MAP option is carried in the Router Advertisement (RA) message. Major fields in a MAP option include the IP address of the MAP, Distance (initial value $=1$ ), and Valid lifetime for the MAP option.

Upon reception of an RA with the MAP option, the receiving router must copy the option and re-send it after incrementing the Distance field by one. If the receiving router is also a MAP, it must send its own option together with the received option in the same RA. In this way, an $\mathrm{MH}$ receives MAP options by RA periodically and selects the one with largest value in the Distance field (i.e. the highest MAP) as its MAP. After selecting the MAP, the MH can bind its current location (on-link CoA or $L C O A$ obtaining from the access router) with the address of the selected MAP (Regional CoA or $R C o A)$. The MAP will receive all packets on behalf of the MH it is serving and will encapsulate and forward them directly to the MH's current address. If the mobile node changes its current address (LCoA) within a local MAP domain, it only needs to register the new address with the MAP. Hence, only the RCoA needs to be registered with correspondent nodes and the HA.

The default MAP selection scheme in HMIPv6 that is also called distance-based MAP selection reduces the number of RCoA Binding Updates $(B U)$ to the HA, but it results in higher load to the higher MAPs. Some methods have been proposed to improve the default MAP selection scheme. A MAP selection scheme based on speed estimation is proposed in [9], in which the speed of an $\mathrm{MH}$ is estimated by measuring

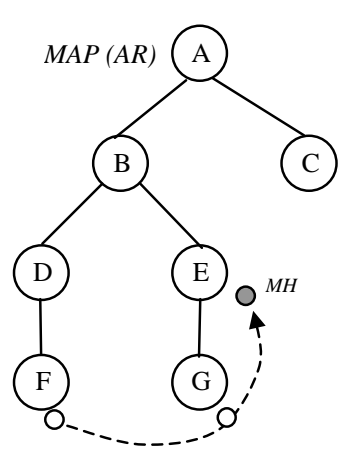

\begin{tabular}{|c|c|c|c|}
\hline MAP ID & Distance & Lifetime & Total \\
\hline \multirow{3}{*}{ A } & 4 & 8 & \multirow{3}{*}{3} \\
\hline & 4 & 9 & \\
\hline & 3 & 10 & \\
\hline \multirow{3}{*}{ B } & 3 & 8 & \multirow{3}{*}{3} \\
\hline & 3 & 9 & \\
\hline & 2 & 10 & \\
\hline $\mathrm{D}$ & 2 & 8 & 1 \\
\hline \multirow{2}{*}{$\mathrm{E}$} & 2 & 9 & \multirow[b]{2}{*}{2} \\
\hline & 1 & 10 & \\
\hline $\mathrm{F}$ & 1 & 8 & 1 \\
\hline $\mathrm{G}$ & 1 & 9 & 1 \\
\hline
\end{tabular}

Figure 2. An example of MAP Option Table

the frequency of LCoA BU to the MAP. A higher frequency of LCoA binding update implies a higher speed, and a faster $\mathrm{MH}$ selects a higher MAP in the scheme. However, since the moving range of an $\mathrm{MH}$ is not considered in the scheme of speed estimation, the scheme fails to select an appropriate MAP for the MH with a high speed but small roaming area.

A load balancing mobility management called Distributed Location Management $(D L M)$ is proposed in [10], in which a threshold of hop count $\left(N_{h}\right)$ is defined such that an $\mathrm{MH}$ can roam among nearby MAPs within $N_{h}$ hops without reselecting a new MAP. The DLM scheme reduces the signaling cost of RCoA binding udpate and achieves load balancing to some extent at the cost of longer delivery path.

\section{Moving AreA-BASEd MAP SELECTION}

The proposed scheme is called Moving Area-Based MAP selection, in which the MH keeps track of its moving area to determine the best MAP. Figure 1 illustrates the basic idea of the proposed scheme, in which each mobile host selects the closest (lowest) MAP that covers its moving area. In this way, each MH selects a proper MAP (rather than the most distant/highest one) that fits for its moving behavior.

The mechanism for a $\mathrm{MH}$ to keep track of its moving area is based on the MAP option periodically transmitted downward by each MAP in the hierarchy. The MH records the total number of MAP options issued by each MAP in its MAP Option Table. When the MH moves to a new subnet, it invokes the MAP selection algorithm to select the MAP with most MAP options received by the MH over a predefined period of time. If there are two or more MAP candidates with the same most MAP options, the MH selects the lowest MAP.

For example, the MH in Figure 2 moves from MAP $F$ (the initial position) to $M A P G$, and then to $M A P E$ as displayed on the left side in the figure. We assume the MH receives only one RA (sent out by MAP A) while it is on each MAP and the lifetime for a new MAP option is set to 10 moves. The MAP Option Table maintained by the $\mathrm{MH}$ is shown on the right side of the figure. According to the proposed scheme, MAP $B$ is selected as the new MAP by the MH since it is the closest 


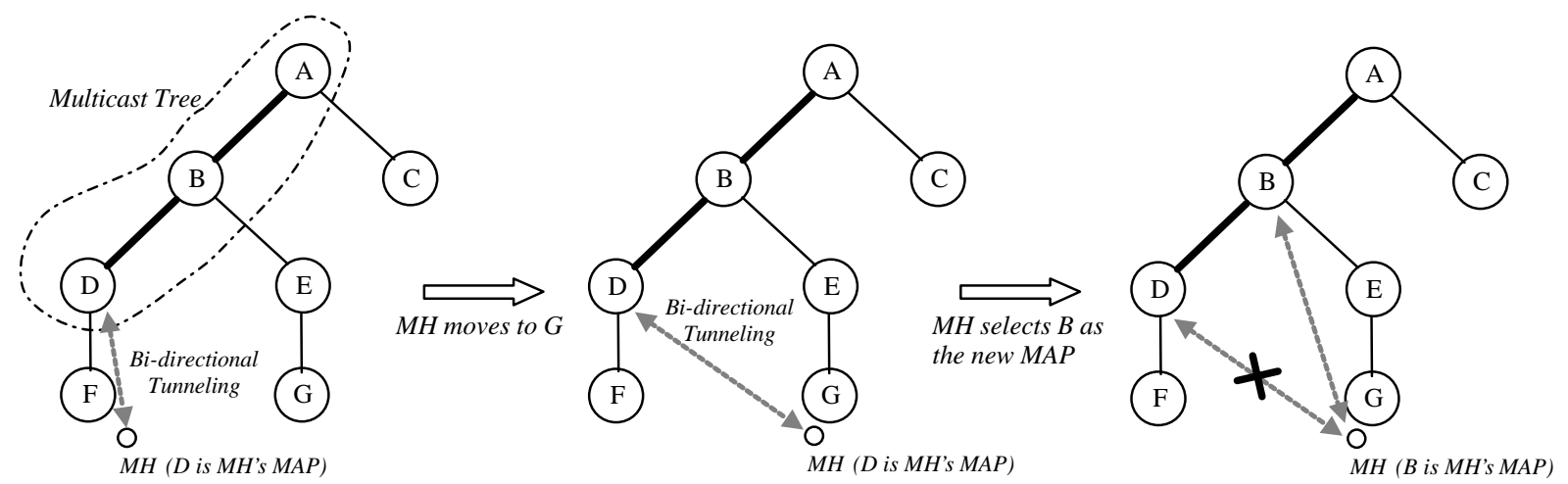

Figure 3. E.g. Multicast Data Delivery

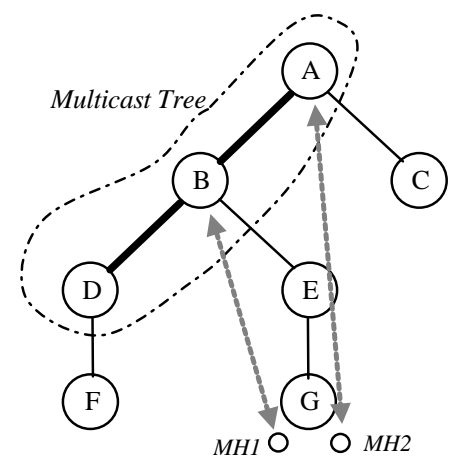

Bi-directional Tunneling

$M H 1$ 's $M A P=B, M H 2$ 's $M A P=A$

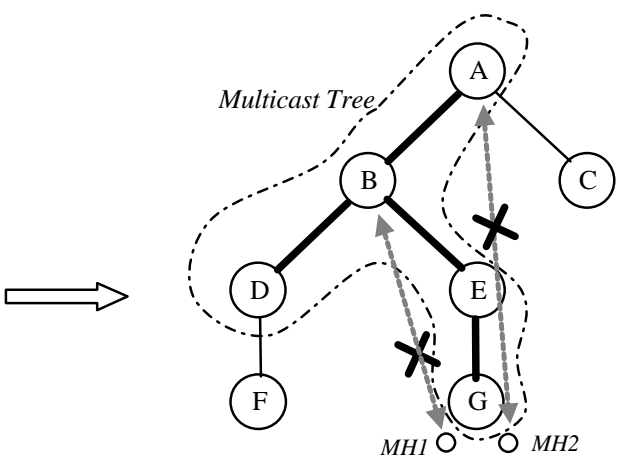

1. G detects that more than two group members are in the subnet, so $G$ joins the multicast tree.

2. MH1 and MH2 inform their MAPs to stop tunneling packets.

(a) Tunnel Convergence problem

(b) Solution for the problem

Figure 4. Tunnel Convergence problem and its solution

MAP from which the MH has received the most MAP options.

\section{Multicast Extension of HMIPv6}

The proposed multicast scheme is basically an improvement of Remote Subscription (RS) applied in HMIPv6. Operations of a $\mathrm{MH}$ after handoff to a new subnet in the proposed multicast scheme are explained in the following. When the MH arrives at a new subnet, it will first obtain a stateless auto-configuration address as its LCoA from the access router. If the subnet is already on the multicast tree of the desired group, the MH rejoins the multicast group locally by sending a Multicast Listener Discovery (MLDv2) report message. In the mean time, the MH sends a BU to its MAP with its current LCoA and a MLDv2 report message to inform its MAP not to forward multicast packets by tunneling.

On the other hand, if the arrived subnet is not on the multicast tree, the MH sends a BU with its new LCoA to its MAP. The MAP tunnels multicast packets to the MH. Moreover, if a new MAP is selected by the MAP selection algorithm, in addition to sending a LCOA BU to the new MAP, the MH also sends out an MLDv2 report message asking the new MAP to join the desired multicast group. When $\mathrm{MH}$ receives multicast packets from its new MAP, it notifies the previous MAP to stop tunneling multicast packets to the MH. An example of multicast data delivery in the proposed scheme is displayed in Figure 3.

MAP-based tunneling in the proposed multicast protocol may results in the problem of tunnel convergence when two or more MAPs tunnels the multicast packets of the same group to different MHs that resides in the same subnet as displayed in Figure 4-(a). In order to solve the tunnel convergence problem and reduce redundancy in packet transmission, the MAP that is the convergence point of tunneling must be able to detect the problem. The MLDv2 specific query message is used for a MAP to find out the members (MHs) of a specific group in the subnet. Upon receiving the specific query message, each $\mathrm{MH}$ must sends an MLDv2 report message to the MAP when the $\mathrm{MH}$ has joined or is joining the specific group. If the MAP receives more than two MLDv2 report messages for a same group, which implies the tunneling convergence problem of the group is forming, the MAP joins the multicast tree of the 


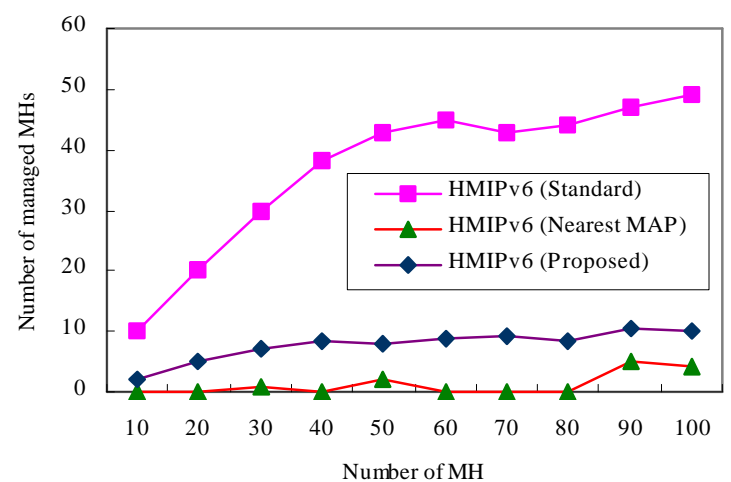

Figure 5. Number of managed MHs in the root and first level MAPs

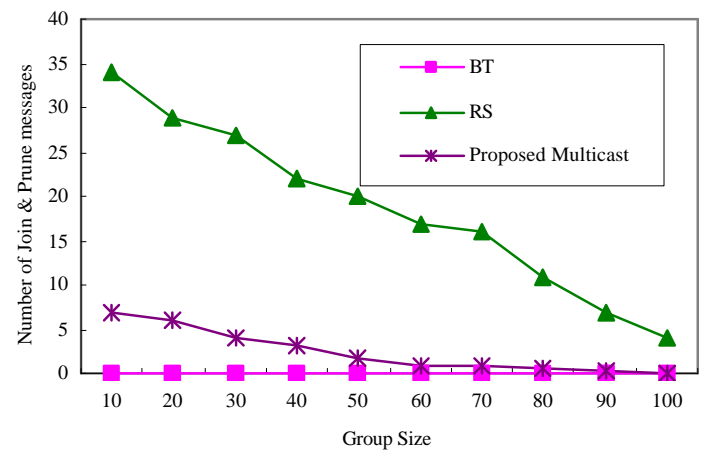

Figure 7. Multicast tree maintenance cost

group and each member of the group in the subnet asks their MAP to stop tunneling packets. As illustrated in Figure 4-(b), $M A P G$ detects the tunnel convergence problem and joins the multicast tree. Meanwhile, MH1 and MH2 inform their MAPs to stop tunneling packets respectively.

\section{Performance Evaluation}

\section{A. Simulation environment}

The network model in the simulation consists of 341 randomly generated nodes. Each node represents a MAP with access router and multicast routing function that in charge of a subnet. Wired links are added among the 341 nodes to form a 5-level and 4-ary hierarchy of MAPs. The number of MAPs in the hierarchy in each level is as follows: level 0 (root) $=1$ MAP, level $1=4$ MAPs, level $2=16$ MAPs, level $3=64$ MAPs, level $4=256$ MAPs. A HA that is 4-hop away from the root MAP is added beyond the hierarchy. The HA is the home agent for all mobile hosts in the network.

The number of mobile hosts varies from 10 to 100 in the simulation, and initial locations for the mobile hosts are randomly selected from the MAPs (subnets). Time is slotted in the simulation, and in the beginning of a new time slot, $\mathrm{MH}$ can either stay in the same subnet or move to any of the neighboring subnets with equal probability. To simulate multicast transmission, a single and static multicast source is

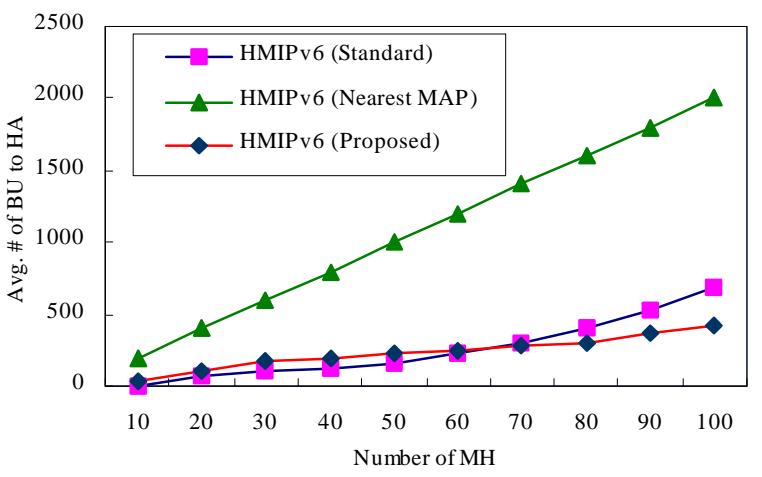

Figure 6. Number of binding updates to HA

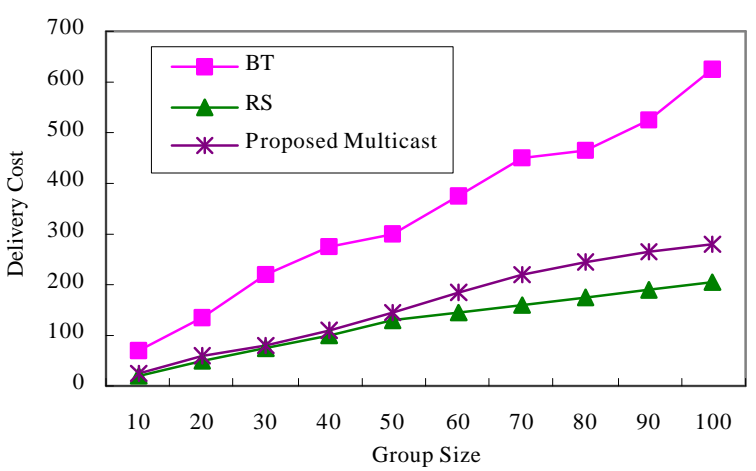

Figure 8. Average delivery cost per packet

located in the root MAP

\section{B. Simulation results}

Figure 5 shows that the load (the average number of MHs managed by MAP) of highers MAP (root and level 1 MAPs) is reduced in the proposed Moving Area-based MAP selection scheme, and as shown in Figure 6, the signaling cost (the average number of binding update sent to the $H A$ ) of the proposed scheme is only a little bit larger than the standard scheme.

Figure 7 shows the average number of control messages (including join and prune messages) for multicast tree maintenance in the proposed multicast protocol. Since the multicast tree in BT (Bi-directional Tunneling) remains unchanged regardless of the movement of mobile hosts, BT generates no control messages for multicast tree maintenance in equilibrium state. RS (Remote Subscription) generates the most signaling cost for maintaining the multicast tree, since multicast tree in RS is subject to change whenever a mobile host moves to a new subnet. In a word, the signaling cost for multicast tree maintenance in the proposed scheme is significantly smaller than that of the original RS scheme, which means the multicast tree constructed by the proposed scheme is relatively stable.

The average multicast delivery cost for each scheme is displayed in Figure 8, in which the average delivery cost of 


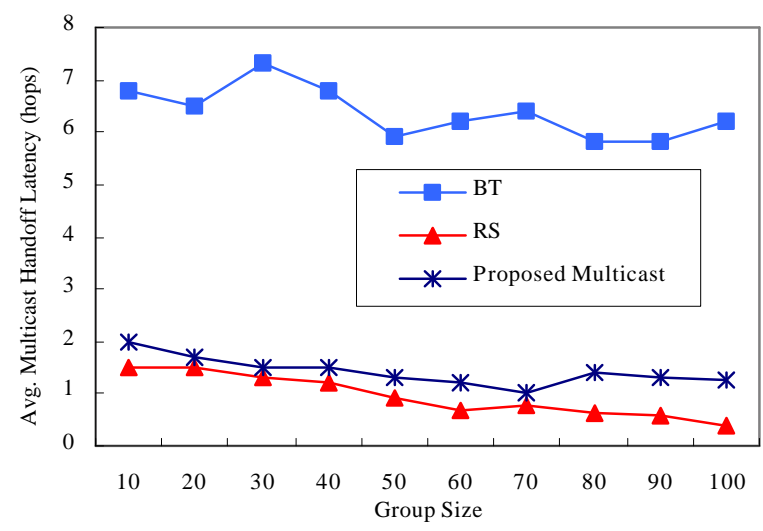

Figure 9. Avg. Handoff Latency for Multicasting

proposed multicast scheme is pretty close to that of RS (the optimal case). The result demonstrates the efficiency of multicast routing of the proposed scheme.

The average handoff latency (in hops) for multicasting is displayed in Figure 9, in which we define the handoff latency for multicasting as the duration from the handoff of a mobile host to the time when the $\mathrm{MH}$ receives the first multicast packet in the new subnet. Since the MH receives multicast packets forwarded by its MAP, which is typically close to the current location of the $\mathrm{MH}$, the proposed multicast scheme only results in a moderate increase in the average handoff latency for multicasting over RS.

\section{CONCLUSION}

To reduce the signaling cost and enhance seamless handoff of MIPv6, a hierarchical architecture for supporting local registration- Hierarchical Mobile IPv6 (HMIPv6) was proposed. To provide efficient local mobility management, a multilevel hierarchy of MAPs (Mobile Anchor Point) in HMIPv6 is recommended. The default MAP selection algorithm specified in the draft of HMIPv6 is based on selecting the MAP that is most distant to avoid frequent re-registrations. However, the default MAP selection algorithm apparently results in heavy load in higher MAPs as well as a longer tunneling path between selected MAP and $\mathrm{MH}$. By considering the moving area of a mobile host, a more efficient MAP selection scheme is proposed in the paper.

Moreover, given that few research papers discussing the multicast extension of HMIPv6, an efficient multicast extension of HMIPv6 integrating the MAP selection algorithm is also proposed in the paper. The proposed multicast scheme is basically an improvement of Remote Subscription (RS) applied in HMIPv6. Simulation study shows that the average number of control messages for multicast tree maintenance is significantly smaller than that of the original RS scheme, which means the multicast tree constructed by the proposed scheme is relatively stable. Moreover, the average delivery cost of proposed multicast scheme is close to that of RS (the optimal case).

\section{REFERENCES}

[1] D. Johnson, C. Perkins, and J. Arkko, "Mobility support in IPv6," IETF, RFC 3775, Jun. 2004.

[2] H. Soliman, C. Castelluccia, K. El-Milki, and L. Bellier, "Hierarchical Mobile IPv6 mobility management (HMIPv6)," Internet Draft, Dec. 2004, IETF. draft-ietf-mipshop-hmipv6-04.txt (work in progress)

[3] R. Vida, and L. Costa, "Multicast Listener Discovery Version 2 (MLDv2) for IPv6" IETF, RFC 3810, Jun. 2004.

[4] T. Harrison, and C. williamson, "Mobile Multicast (MoM) protocol: Multicast support for mobile hosts," Proceedings, ACM MobiCOM'97, pp151-160.

[5] Chunhung Richard Lin and Kai-Min Wang, "Mobile multicast support in IP networks," Proceedings, IEEE INFOCOM, 2000, pp1664-1672.

[6] H.-S. Shin and Y.-J. Suh, "Multicast Routing Protocol in Mobile Networks"; Proceedings, IEEE International Conference on Communications (ICC), 2000, pp.1416-1420.

[7] Y.-J. Suh, D.-H. Kwon, and W.-J. Kim, "Multicast Routing by Mobility Prediction for Mobile Hosts," Proceedings, IEEE International Conference on Communications (ICC), 2003, pp. 865-869.

[8] J.-R. Lai, Wanjiun Liao, M.-Y. Jiang, and C.-A. Ke, "Mobile Multicast with Routing Optimization for Recipient Mobility," Proceedings, IEEE International Conference on Communications (ICC), 2001, pp. 1340-1344.

[9] K. Kawano, K. Kinoshita, and K. Murakami, "A Multilevel Hierachical Distributed IP Mobility Management Scheme for Wide Area Networks," Proceedings, International Conference on Computer Communications and Networks (ICCCN), 2002, pp. $480-484$.

[10]M. Bandai and I. Sasase, "A Load Balancing Mobility Management for Multilevel Hierarchical Mobile IPv6 Networks," Proceedings, IEEE International Symposium on Personal Indoor and Mobile Radio Communications (PIMRC), vol.1, 2003, pp. 460-464. 